\title{
Correlation between Interleukin-6 (IL-6), High Sensitivity C-Reactive Protein (hsCRP), Endothelin-1 (ET-1), Asymmetric Dimethylarginine (ADMA) and Insulin Resistance (HOMA-IR) in Central Obese Men
}

\author{
Andri Hidayat ${ }^{1,2}$, Andi Wijaya ${ }^{1,2}$, Harun Alrasyid ${ }^{3}$
}

${ }^{1}$ Prodia Clinical Laboratory, Jl. Letjen. S. Parman No.17/223G, Medan, Indonesia

${ }^{2}$ Post Graduate Program in Clinical Biochemistry, Hasanuddin University, Jl. Perintis Kemerdekaan Kav.10, Makassar, Indonesia ${ }^{3}$ Faculty of Medicine, University of Sumatera Utara,Jl. Dr. Mansyur No.5, Medan, Indonesia *Correspondence: andri.hidayat@prodia.co.id

\section{Abstract}

$\mathrm{B}$ ACKGROUND: Many studies have shown that obesity was closely related to insulin resistance via several pathways such as inflammation, oxidative stress, lipolysis, and endothelial dysfunction. This study was carried out to observe the correlation between inflammation (IL-6 and hsCRP), lipolysis process (ET-1), and endothelial dysfunction (ADMA) and insulin resistance (HOMA-IR) in centrally obese men.

METHODS: This was a cross sectional study on 62 male subjects aged 30-60 years old with waist circumference (WC) $>90 \mathrm{~cm}$. IL-6, ET-1 and ADMA levels were measured using ELISA method, while hsCRP and insulin were measured using chemiluminescence method. All blood testings were conducted in Prodia Clinical Laboratory.

RESULTS: The results showed that WC was significantly correlated with hsCRP $(r=0.294, \mathrm{p}=0.022)$, ET-1 $(\mathrm{r}=$ $0.257, \mathrm{p}=0.047)$, and ADMA $(\mathrm{r}=0.338, \mathrm{p}=0.009)$. We also found a significant correlation between hsCRP with HOMA-IR $(r=0.324 \mathrm{p}=0.021)$, ADMA with HOMA-IR $(\mathrm{r}=0.280 \mathrm{p}=0.045)$, and IL-6 with hsCRP $(\mathrm{r}=0.437, \mathrm{p}$ $=0.003)$.

CONCLUSIONS: hsCRP and ADMA have significant correlation with HOMA-IR in centrally obese men. HOMA-IR significantly increases in subjects with ADMA above median and either IL- 6 or hsCRP above median, as compared to those in the other groups. Inflammation and endothelial dysfunction are important causal pathways of insulin resistance state in centrally obese men.

KEYWORDS: Obesity, IL-6, hsCRP, ET-1, ADMA, HOMA-IR.

Indones Biomed J 2011; 3(1): 43-50

\section{Introduction}

Obesity plays a key role in the dramatic increase of type 2 diabetes mellitus (T2DM) and cardiovascular disease (CVD) worldwide as well as in Indonesia where the prevalence of obesity has increased from $13.13 \%$ in 2004 to $19.1 \%$ in 2007 (1). 
Central obesity is associated with the state of chronic, low-grade inflammation, particularly in white adipose tissue. Adipose tissue is characterized by macrophage infiltration and that these macrophages are an important source of inflammation in the tissue by over expressing several proinflammatory adipokines such as tumor necrosis factor alpha (TNF- $\alpha$ ) and interleukin-6 (IL-6) $(2,3,4,5)$.

Evidence showed that IL-6 levels were $30 \%$ higher in obese patients as compared with that in healthy individuals. Apart from the proinflammatory properties, IL-6 is positively associated with fat mass, insulin resistance, and free fatty acid (FFA) levels $(6,7)$.

C-reactive protein (CRP) is an acute phase reactant produced in hepatocytes in response to stimulation of IL-6. CRP with its inflammatory properties having been identified as a risk factor for developing T2DM. Circulating plasma CRP level was elevated in obese subjects and associated with the amount of body fat, as assessed anthropometrically by body mass index (BMI), and with visceral obesity (assessed by high WC) and individuals with the metabolic syndrome and on insulin resistance $(6,7)$.

The release of FFA from adipocytes (lipolysis) increased in obese state and is well known to play a major role in the development of obesity-associated complications. Elevated circulating levels of FFA can impair insulin signaling pathways and further induced oxidative stress, inflammation, vascular endothelial reactivity, and insulin resistance. Lipolysis increases FFA levels, and inflammation and insulin resistance can form a viscious cycle $(5,8,9)$.

Fat cells are surrounded by stroma vascular cells in which endothelial cells are also present. One of the proteins secreted by the endothelial cell is endothelin (ET), which is a potent vasocontrictor and involved in the regulation of blood pressure. ET-1 attenuates the inhibiting effect of insulin on lipolysis in the viscerals but not in the subcutaneous fat cells via binding to ET receptor A $\left(E_{A} R\right)$ and $B\left(E T_{B} R\right)$. Previous studies have shown that ET-1 levels increased in subjects with obesity and T2DM $(8,10,11)$.
In obesity the plasma concentration of ADMA is elevated and ADMA inhibits the activity of endothelial Nitric Oxide Synthase (eNOS), thus reducing the synthesis and secretion of nitric oxide (NO), whereas the imbalance between production of $\mathrm{NO}$ and secretion of ET-1 leads to decreased blood flow, thus further worsens insulin resistance $(6,12)$.

\section{Materials and Methods}

This was an observational study with a cross-sectional design using 62 central obese men having WC $>90 \mathrm{~cm}$ and aged 30-60 years. The parameters measurements were performed in Prodia Clinical Laboratory, Medan. Subjects having hsCRP levels $>10 \mathrm{mg} / \mathrm{L}$, impaired renal function (GFR < $60 \mathrm{~mL} / \mathrm{min}$ ), consumed antiinflammatory, antihypertensive, and antioxidant agents at least 3 months prior to the blood testing were excluded from the study. IL-6 (Quantikine Human IL-6 immunoassay, Cat.No.D6050, R\&D System Inc.), ET-1 (Human Endothelin-1 Immunoassay, Cat.No.BBE5, R\&D System Inc.) and ADMA (ADMA-ELISA, Cat.No.17-EA201/96, DLD Diagnostika GMBH) levels were measured using ELISA method, while hsCRP (hsCRP, Cat.No.LKCRP1, Siemens Diagnostics), and insulin (Insulin, Cat. No.L2K1N2, Siemens Diagnostics) were measured using chemiluminescence method.

\section{Results}

This study was carried out confinedly on adult male subjects to prevent any biases from hormonal effects. The baseline characteristics of the study subject are summarized in Table 1. 
Table 1. Baseline Characteristics of the Study Subjects

\begin{tabular}{lrrrrr}
\hline Variable & Minimum & Maximum & Median & Average & SD \\
\hline Age $(\mathrm{year})$ & 30.00 & 60.00 & 38.91 & 39.59 & 6.88 \\
Height $(\mathrm{cm})$ & 154.00 & 188.00 & 168.00 & 168.02 & 6.20 \\
Weight $(\mathrm{kg})$ & 65.00 & 110.00 & 83.00 & 84.96 & 12.31 \\
BMI $\left(\mathrm{kg} / \mathrm{m}^{2}\right)$ & 24.34 & 38.57 & 29.06 & 30.05 & 3.53 \\
WC $(\mathrm{cm})$ & 91.00 & 118.00 & 98.00 & 101.60 & 7.60 \\
SBP $(\mathrm{mmHg})$ & 100.00 & 130.00 & 117.50 & 114.84 & 9.36 \\
DBP $(\mathrm{mmHg})$ & 70.00 & 85.00 & 80.00 & 78.23 & 4.54 \\
FBG $(\mathrm{mg} / \mathrm{dL})$ & 47.00 & 118.00 & 85.00 & 85.90 & 12.84 \\
hsCRP $(\mathrm{mg} / \mathrm{L})$ & 0.20 & 7.96 & 3.37 & 3.09 & 2.27 \\
Insulin $(\mu \mathrm{lu} / \mathrm{mL})$ & 2.00 & 89.80 & 7.18 & 12.28 & 14.64 \\
HOMA-IR & 0.34 & 4.48 & 1.37 & 1.75 & 0.98 \\
IL-6 $(\mathrm{pg} / \mathrm{mL})$ & 0.41 & 5.73 & 1.34 & 1.76 & 1.19 \\
ET-1 $(\mathrm{pg} / \mathrm{mL})$ & 0.48 & 1.81 & 1.03 & 1.06 & 0.31 \\
ADMA $(\mu \mathrm{mol} / \mathrm{L})$ & 0.26 & 0.85 & 0.58 & 0.59 & 0.12
\end{tabular}

WC $=$ Waist Circumference; BMI $=$ Body Mass Index; SBP = Systolic Blood Pressure; DBP = Diastolic Blood Pressure; FBG = Fasting Blood Glucose; HOMA-IR = Homeostasis Model Assessment - Insulin Resistant; hsCRP = High Sensitivity C-Reactive Protein; ADMA = Asymmetric Dimethylarginine; IL-6 = Interleukin-6; ET-1 = Endothelin-1. The p-value above is the result of Kolmogorov-Smirnov normality test with a degree of confidence used was $95 \%$.

Table 2 shows the results of Pearson and Spearman's correlation analysis between variables. It shows that WC significantly correlated in a linear model with log hsCRP $(\mathrm{r}=0.294)$, ET-1 $(\mathrm{r}=0.257)$, ADMA $(\mathrm{r}=0.338)$ and with HOMA-IR $(r=0.462)$. IL-6 significantly correlated with $\log$ hsCRP $(r=0.437)$. Log hsCRP significantly correlated with HOMA-IR $(\mathrm{r}=0.324)$, ADMA significantly correlated with HOMA-IR $(r=0.280)$ and IL-6 level was found to be positively but not significantly correlated with HOMA-IR $(\mathrm{r}=0.293 ; \mathrm{p}=0.053)$.

Table 2. Correlation Between Parameters

\begin{tabular}{|c|c|c|c|c|c|}
\hline Variable & $\begin{array}{c}\text { IL-6 } \\
r\end{array}$ & $\begin{array}{c}\text { Ig hsCRP } \\
r\end{array}$ & $\begin{array}{c}E T-1 \\
r\end{array}$ & $\begin{array}{c}\text { ADMA } \\
r\end{array}$ & $\begin{array}{c}\text { HOMA-IR } \\
\mathbf{r}\end{array}$ \\
\hline WC" & 0.228 & $0.294^{*}$ & $0257^{*}$ & $0.338^{*}$ & $0.462^{*}$ \\
\hline SBP & -0.080 & 0.027 & 0.051 & -0.118 & 0.031 \\
\hline DBP\# & 0.253 & 0.104 & $0.262^{*}$ & 0.087 & -0.030 \\
\hline IL-6 & 1.000 & $0.437^{*}$ & 0.183 & 0.248 & 0.293 \\
\hline ET-1 & 0.183 & -0.019 & 1.000 & -0.073 & 0.099 \\
\hline ADMA & 0.248 & 0.109 & -0.073 & 1.000 & $0.280^{*}$ \\
\hline HOMA-IR & 0.293 & $0.324^{*}$ & 0.099 & $0.280^{*}$ & 1.000 \\
\hline
\end{tabular}

WC = Waist Circumference; SBP = Systolic Blood Pressure; DBP = Diastolic Blood Pressure; HOMA-IR = Homeostasis Model Assessment - Insulin Resistant; hsCRP = High Sensitivity C-Reactive Protein; ADMA = Asymmetric Dimethylarginine; IL-6 = Interleukin-6; ET-1 = endothelin-1; ${ }^{\star}$ significant with $\mathrm{p}=0.05$ confidence level. All Data are Pearson correlation test results, except for (\#) Spearman test results. 
We categorized the parameters into two groups using median as the cut off value, except hsCRP using $3 \mathrm{mg} / \mathrm{L}$ (medium Risk) and HOMA-IR using 2(13). The correlation between groups was analyzed by crosstabulation test (Table 3). It was shown that ADMA significantly correlated with HOMA-IR ( $\mathrm{p}=0.015$; odds ratio $=3.87$ ), IL-6 not significantly correlated with HOMA-IR ( $\mathrm{p}=0.093$; odds ratio $=2.667)$, hsCRP not significantly correlated with HOMA-IR ( $p=0.110$; odds ratio $=2.33$ ), and ET-1 not significantly correlated with HOMA-IR $(\mathrm{p}=0.610$; odds ratio $=1.313$ ).

\section{Table 3. Crosstabulation analysis between IL-6, hs-CRP, ET-1 and ADMA in Subjects with HOMA-IR $\geq 2$ and Subjects with HOMA-IR $\leq \mathbf{2}$}

\begin{tabular}{|c|c|c|c|c|c|c|c|c|c|}
\hline \multirow[t]{2}{*}{ Variable } & & \multicolumn{2}{|c|}{$\begin{array}{l}\text { HOMA-IR } \\
\geq 2\end{array}$} & \multicolumn{2}{|c|}{$\begin{array}{c}\text { HOMA-IR } \\
<2\end{array}$} & \multirow[t]{2}{*}{ p } & \multirow[t]{2}{*}{ OR } & \multicolumn{2}{|c|}{ Cl $95 \%$} \\
\hline & & N & $\%$ & N & $\%$ & & & Min & $\operatorname{Max}$ \\
\hline \multirow[t]{2}{*}{ ADMA } & $\geq$ Med & 16.0 & 55.2 & 13.0 & 44.8 & $0.015^{*}$ & 3.868 & 1.260 & 11.880 \\
\hline & $<$ Med & 7.0 & 24.1 & 22.0 & 75.9 & - & - & - & - \\
\hline \multirow[t]{2}{*}{ IL-6 } & $\geq$ Med & 15.0 & 60.0 & 10.0 & 40.0 & 0.093 & 2.667 & 0.850 & 8.366 \\
\hline & $<$ Med & 9.0 & 36.0 & 16.0 & 64.0 & - & - & - & - \\
\hline \multirow[t]{2}{*}{ hsCRP } & $\geq 3$ & 14.0 & 53.8 & 12.0 & 46.2 & 0.110 & 2.333 & 0.827 & 6.580 \\
\hline & $>3$ & 12.0 & 33.3 & 24.0 & 66.7 & - & - & - & - \\
\hline \multirow[t]{2}{*}{ ET-1 } & $\geq \operatorname{Med}$ & 14.0 & 46.7 & 16.0 & 53.3 & 0.610 & 1.313 & 0.472 & 3.653 \\
\hline & $<$ Med & 12.0 & 40.0 & 18.0 & 60.0 & - & - & - & - \\
\hline
\end{tabular}

HOMA-IR = Homeostasis Model Assessment - Insulin Resistant; OR = Odds Ratio; CI = Confidence Interval; ADMA = Asymmetric Dimethylarginine; IL-6 = Interleukin-6; hsCRP = High Sensitivity C-Reactive Protein; ET-1 = Endothelin-1.

Table 4 summarizes the results of logistic regression analysis, with preliminary data analyzed are ADMA, ET-1, IL-6 and hsCRP. We found that ADMA, hsCRP and IL-6 played an important role in the patophysiology of insulin resistance.

Table 4. Regression Analysis of ADMA, hsCRP, IL-6 (dependent variable: HOMA-IR)

\begin{tabular}{|c|c|c|c|c|c|c|c|}
\hline & \multirow[t]{2}{*}{$\beta$} & \multirow[t]{2}{*}{ SE } & \multirow[t]{2}{*}{ Wald } & \multirow{2}{*}{$\begin{array}{c}\mathbf{p} \\
\text { Value }\end{array}$} & \multirow{2}{*}{$\begin{array}{c}\text { Exp } \\
(\beta)\end{array}$} & \multicolumn{2}{|c|}{$\mathrm{Cl} 95 \%$} \\
\hline & & & & & & Low & High \\
\hline ADMA & 2.296 & 0.785 & 8.545 & 0.003 & 9.932 & 2.131 & 46.295 \\
\hline hsCRP & 1.329 & 0.778 & 2.920 & 0.087 & 3.778 & 0.823 & 17.350 \\
\hline IL-6 & 1.235 & 0.729 & 2.872 & 0.090 & 3.437 & 0.824 & 14.335 \\
\hline Constant & -2.534 & 0.853 & 8.824 & 0.003 & 0.079 & - & - \\
\hline
\end{tabular}

SE $=$ standard errors; $\operatorname{Exp}(\beta)=$ odds ratios; $\mathrm{Cl}=$ Confidence Interval; HOMA-IR $=$ Homeostasis Model Assessment Insulin Resistant; OR = Odds Ratio; $\mathrm{Cl}=$ Confidence Interval; ADMA = Asymmetric Dimethylarginine; IL-6 = Interleukin-6; hsCRP = High Sensitivity C-Reactive Protein; ET-1 = Endothelin-1. 
As seen in Table 5, we combined the parameters to see how the synergism of inflammation (IL-6 and CRP) and endothelial (ADMA) pathways could affect the insulin resistance (HOMA-IR). The results showed that most subjects who have HOMA-IR $>2$ had higher ADMA level, combined with either high $\mathrm{IL}-6$ or CRP level $(69.23 \%)$ (Figure 1).

Table 5. Crosstabulation Analysis for Correlation between Combined of IL-6, hsCRP, ADMA with HOMA-IR

\begin{tabular}{lrrr}
\hline & HOMA $\geq 2$ & HOMA $<2$ & Total \\
\hline One of IL-6, CRP $\geq$ Med \& ADMA $\geq$ Med & $9(69.23 \%)$ & $4(30.77 \%)$ & $13(100 \%)$ \\
One of IL-6, CRP $\geq$ Med \& ADMA < Med & $3(21.43 \%)$ & $11(78.57 \%)$ & $14(100 \%)$ \\
Both of IL-6, CRP $\geq$ Med \& ADMA $\geq$ Med5 & $(55.56 \%)$ & $4(44.44 \%)$ & $9(100 \%)$ \\
Both of IL-6, CRP $\geq$ Med \& ADMA < Med & $3(42.86 \%)$ & $4(57.14 \%)$ & $7(100 \%)$ \\
Both of IL-6, CRP < Med \& ADMA $\geq$ Med & $2(28.57 \%)$ & $5(71.43 \%)$ & $7(100 \%)$ \\
Both of IL-6, CRP < Med \& ADMA < Med & $1(12.50 \%)$ & $7(87.50 \%)$ & $8(100 \%)$ \\
TOTAL & $23(39.66 \%)$ & $35(60.34 \%)$ & $58(100 \%)$ \\
\hline
\end{tabular}

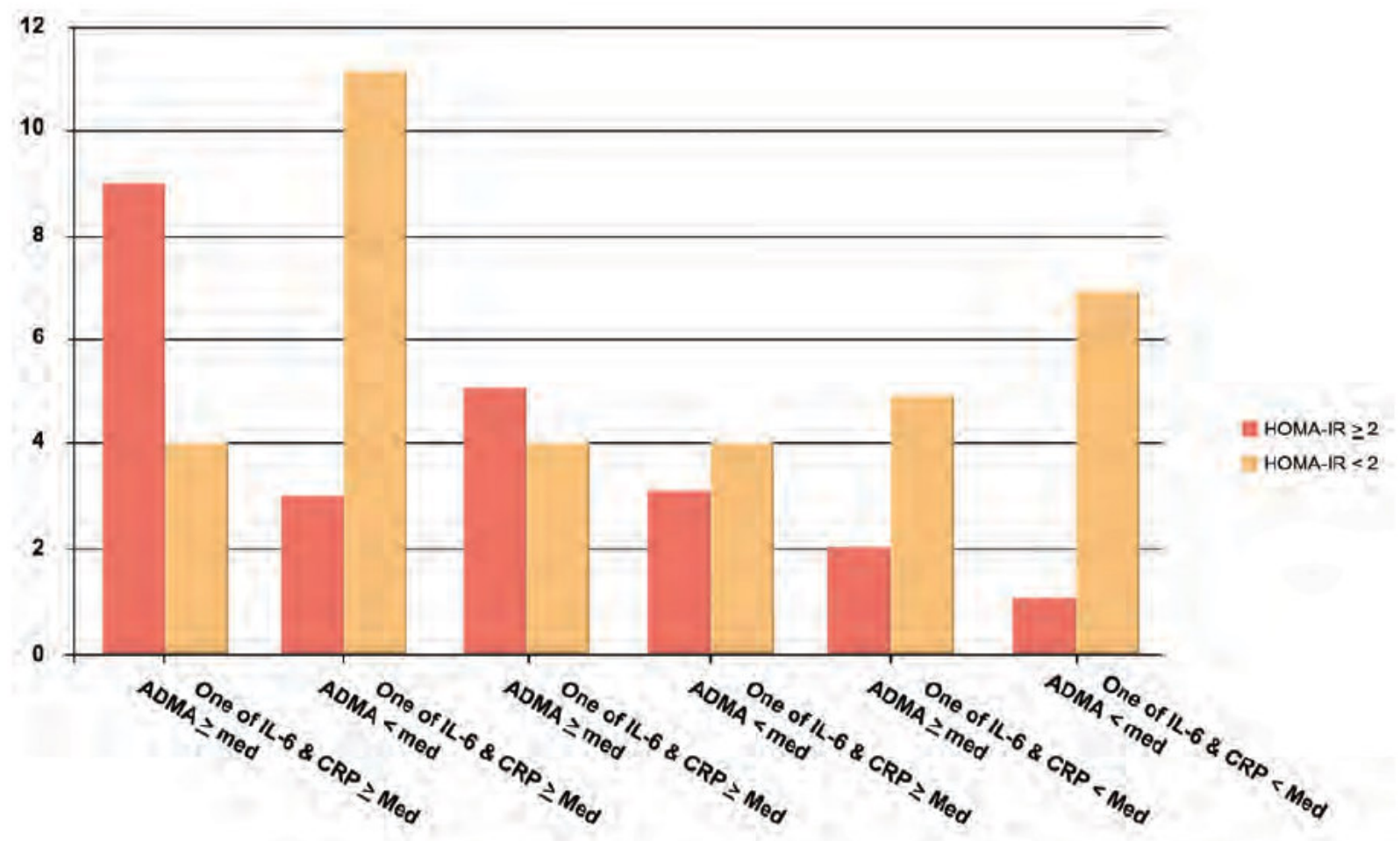

Figure 1. Correlation between Combined IL-6, hsCRP, and ADMA with HOMA-IR. 


\section{Discussion}

\section{IL-6 and hsCRP with insulin resistance (HOMA-IR)}

The molecularmechanismunderlying IL-6 mediated insulin resistance could involve activation of proinflammatory kinases, suppressor of cytokine signaling 3 (SOCS3) and phosphatases. These molecules appeared to inhibit insulin signaling either by interfering with insulin receptor substrate 1 (IRS-1) and IRS- 2 tyrosine phosphorylation or by targeting IRS-1 and IRS- 2 for proteosomal degradation (2).

In this study, IL-6 level was found to be positively but not significantly correlated with HOMA-IR; and hsCRP was significantly correlated with HOMA-IR. Our study confirmed the finding of Kristiansen et al. which showed that CRP was a better predictive marker for T2DM, whereas the study by Pradhan et al. found that CRP had a better correlation with T2DM compared to IL-6, due to a longer hsCRP half life than IL-6 $(14,15)$.

There still exist many controversies regarding the correlation between IL- 6 and insulin resistance. Some studies showed IL-6, not TNF- $\alpha$, was released rapidly into the circulation after exercise. This is paradoxal in terms of the fact that on one hand IL-6 is markedly produced and released in post-exercise period when insulin action is enhanced, while on the other hand IL- 6 has been associated with obesity and reduced insulin action. A study by Glund et al. showed that IL-6 caused 1.5 fold increase of glucose transport in human skeletal muscles. IL-6 directly increases glucose uptake and metabolism and enhances insulin sensitivity in skeletal muscles $(16,17)$.

Another study of Vazquez et al. worked on the hypothesis that the effects of IL- 6 on skeletal muscle cells may depend on the duration of exposure. In the short term (3 hours), IL-6 activates AMP-Activated Protein Kinase (AMPK) without affecting AKT phosphorylation by insulin, while the mechanism by which long-term IL-6 treatment causes impairment of insulin signaling at the IRS-1 level by activation of JNK $1 / 2$, expression of SOCS-3 and activation of Protein Tyrosine Phosphatase 1B (PTP1B) (18).

Madsen $e$ al. concluded that TNF- $\alpha$ induced insulin resistance associated with increased IL-18 gene expression in muscle tissue through activation of NF- $x \mathrm{~B}$, indicating that TNF- $\alpha$ and IL-18 interact with each other and both may have important regulatory roles in the pathogenesis of insulin resistance through activated JNK and IRS-1 phosphorylation, whereas IL-6 does not induce insulin resistance and has no effect on IL-18 gene expression (19).
Our data showed that 36 or $58.1 \%$ of the study subjects were aged $30-40$ years, 47 or $75.81 \%$ of the study subjects had IL- $6<4 \mathrm{pg} / \mathrm{mL}$, suggesting that inflammation in the subjects was still in the early phase and not chronic enough to cause any effect on insulin sensitivity (HOMAIR) (20).

\section{ET-1 and Insulin Resistance}

A recent study has shown that ET-1 level increased up to 2.5 times in obese individuals compared with that in lean individuals (11). Our study also showed ET-1 had a significant positive correlation with WC.

A study by Wilkes et al. showed that ET-1 could stimulate GLUT4 translocation and glucose transport in 3T3-L1 adipose, and this effect was mediated through G q/11 activation and phosphoinositide 3-kinase (PI3K) stimulation. It is well known that chronic ligand stimulation often leads to desensitization of insulin action pathway producing a stage of cellular insulin resistance (21).

An in vitro study by Hermelen et al. showed that ET-1 produced locally in adipose tissue or derived from circulation might be a major factor underlying the selective resistance of visceral adipose tissue to the antilipolytic effect of insulin and might provide a vascular link between visceral fat accumulation and insulin resistance. Only visceral adipocytes were sensitive to ET-1, which occurred after a long-term treatment, so this "anti-insulin" signal is much stronger in visceral than in subcutaneous adipocytes. Eriksson $\mathrm{etal}$. showed that long-term incubation of human adipose tissue with ET-1 could increase lipolytic process significantly $(8,11)$.

Lteif et al. found that the increase of ET-1 has no effects on the whole body glucose uptake, but in vascular smooth muscle cells and adipocytes, ET-1 causes insulin resistance by a PKC-dependent mechanism, other that the vasoconstriction effects of ET-1 while the net tissue actions of insulin in muscle depend in part on the capacity of the vasodilatation of vasculature in response to insulin. In other words, the increase of ET-1 can impede insulin activity (22).

The positive correlation between ET-1 and HOMAIR and WC in this study showed that the level of ET-1 in the study subjects was not high enough to affect insulin sensitivity as concluded from the previous studies by Hermelen, Eriksson and Lteif that chronic elevation of ET-1 was required to affect lipolytic process or to cause insulin resistance.

\section{ADMA and Insulin Resistance}

By t-test we found a significant difference between ADMA level in obese individuals and that in non-obese 
individuals. ADMA level and WC in this study was found to be significantly positively correlated, but not all subjects had a high level of ADMA. We concluded that production and degradation of ADMA in the study subjects were still in a balanced condition.

The correlation between ADMA and HOMA-IR was found to be significantly positive because ADMA was a competitive inhibitor of NOS in L-arginine binding. This means that the increased level of ADMA can impair NO synthesis that further induces endothelial dysfunction, and that endothelial dyfunction is a condition strongly correlated with insulin resistance $(6,23)$.

\section{IL-6, hsCRP, ET-1 and ADMA with Insulin resistance}

The association between obesity and insulin resistance may likely represent a cause and effect relationship since human and animal studies indicate that weight loss/gain correlates closely with increase/decrease in insulin sensitivity, respectively obesity-associated insulin resistance can be explained by the existance of competition between increased circulating Fas and glucose for oxidative metabolism in insulin-responsive cells (13).

Obesity-associated changes in secretion of adipokines such as TNF- $\alpha$, plasminogen activator inhibitor-1 (PAI-1), and IL-6 that modulate insulin signal and increase accumulation of adipose tissue macrophages (ATMs), increase production of cytokines that inhibits insulin signaling (13).

Obesity is a pathologic condition that induces insulin resistance in the early phase by inflammation, endothelial dysfunction, and other metabolites. This study showed the increases of IL-6, ET-1, hs-CRP, and ADMA levels in obese men, in which hs-CRP and ADMA were found to be strongly correlated with HOMA-IR. Exponent $\beta$ from multivariate regression analysis was increased as compared with univariate regression.

The crosstabulation analysis on the correlation between combined IL-6, hs-CRP, ADMA and HOMA-IR showed a significant increase of HOMA-IR in subjects with ADMA above median and either IL-6 or hsCRP above median, compared to those in the other groups. Thus we suggest that in obese individuals, inflammation and endothelial dysfunction have a role in the development of insulin resistance which involves a complex parameters that influence each other.

\section{Conlusions}

Results of this study showed that hsCRP and ADMA were significantly correlated with HOMA-IR in central obese men and that HOMA-IR was significantly increased in subjects with ADMA above 0.58 umol/L and either IL- 6 or hsCRP above median, as compared to the other groups.

\section{Acknowledgement:}

We thank Prodia Education and Research Institute for their funding support of this study.

\section{References:}

1. Depkes RI, Riset Kesehatan Dasar Nasional (RISKESDAS) 2007.

2. Wellen KE, Hotamisligil GS. Inflammation, Stress and Diabetes. $\mathrm{J}$ Clin Invest 2005; 115: 1111-8.

3. Sonnenberg GE, Krakower GR, Kissebah AH. A Novel Pathway to the Manifestations of Metabolic Syndrome. Obes Res 2004; 12 : 180-6.

4. Gustafson B, Hammarstedt A, Andersson CX, Smith U. Inflamed Adipose Tissue: A Culprit Underlying the Metabolic Syndrome and Atherosclerosis. Arterioscler Thromb Vasc Biol 2007; 27: 2276-83.

5. Thalmann TS, Meier CA. Local Adipose Tissue Depots as Cardiovascular Risk Factors. Cardiovasc Res 2007; 75: 690-701.

6. Kim JA, Montagnani M, Koh KK, Quon MJ. Reciprocal Relationships Between Insulin Resistance and Endothelial Dysfunction : Molecular and Pathophysiological Mechanisms. Circulation 2006; 113: 1888-904.

7. Lau DC, Dhillon B, Yan H, Szmitko PE, Verma S. Adipokines : Molecular Links Between Obesity and Atherosclerosis. Am J Physiol Heart Circ Physiol 2005; 288: H2031-41.

8. Eriksson AK, Harmelen V, Stenson BM, Astrom G, Wahlen K, Laurencikiene J, et al. Endothelin-1 Stimulates Human Adipocyte Lipolysis Through The ETA Receptor. Int J Obese 2009; 33: 67-74.

9. Boden G. Free Fatty Acids as Target for Therapy. Curr Opin Endocrinol Diabet 2004; 11: 258-63

10. Williams IL, Wheatcroft SB, Shah AM, Kearney MT. Obesity, Atherosclerosis, and The Vascular Endothelium : Mechanisms Of Reduced Nitric Oxide Bioavailability In Obese Humans. Int J Obes Relat Metab Disord 2002; 26 : 754-64.

11. Harmelen Vv, Eriksson A, Astro G. Vascular Peptide Endothelin-1 Links Fat Accumulation With Alterations of Visceral Adipocyte Lipolysis. Diabetes 2008; 57: 378-86

12. Dandona P,Aljada A, Chaudhuri A, Mohanty P, Garg R. Metabolic Syndrome : A Comprehensive Perspective Based on Interactions Between Obesity, Diabetes and Inflammation. Circulation 2005; 111: 1448-54.

13. Hanley AJG, Williams K, Stern MP., Haffner SM. Homeostasis Model Assessment of Insulin Resistance in Relation to the Incidence of Cardiovascular Disease. Diab Care 2002; 25; 7:1177-84. 
14. Kristiansen OP, Poulsen TM. Interleukin-6 and Diabetes The Good, the Bad, or the Indifferent? Diabetes 2005; 54 :(Suppl. 2): S114-24.

15. Pradhan AD, Manson JE, Rifai N. C-Reactive Protein, Interleukin 6 and Risk of Developing Type 2 Diabetes Mellitus. JAMA 2001; 286: 327-34.

16. Pedersen BK, Febbraio MA. Muscle as an Endocrine Organ : Focus on Muscle-Derived Interleukin-6. Physiol Rev 2008; 88: $1379-406$.

17. Glund S, Deshmukh A, Long YC, Moller T, Koistinen HA, Caidahl K, et al. Interleukin-6 Directly Increases Glucose Metabolism in Resting Human Skeletal Muscle. Diabetes 2007; 56: 1630-37.

18. Vazquez IN, Veledo SF, Alvaro C, Lorenzo M. Dual Role of Interleukin-6 in Regulating Insulin Sensitity in Murine
Skeletal Muscle. Diabetes 2008; 57: 3211-21.

19. Madsen RK, Plomgaard P, Moller K, Mittendorfer B, Pebersen BK. Influence of TNF- $\alpha$ and IL- 6 infusion on insulin sensitivity and expression of IL-18 in humans. Am J Physiol Endocrinol Metab 2006; 291: E108-14.

20. Carey AL, Febbraio MA. Interleukin-6 and insulin sensitivity: friend or foe? Diabetologia 2004; 47: 1135-42.

21. Wilkes JJ, Hevener A, Olefsky J. Chronic Endothelin-1 Treatment Leads to Insulin Resistance In Vivo. Diabetes 2003; 52: 1904-09.

22. Lteif A, Vaishnava P, Baron AD, Mather KJ. Endothelin Limits Insulin Action in Obese/Insulin-Resistant Humans. Diabetes 2007; 56: 728-34.

23. Cooke JP. Does ADMA Cause Endothelial Dysfunction? Arterioscler Thromb Vasc Biol 2000; 20: 2032-7. 